\title{
Performance Evaluation of the WiMAX Network Based on Combining the 2D Markov Chain and MMPP Traffic Model
}

\author{
Tonmoy Saha*, Md. Abu Shufean*, Mahbubul Alam* and Md. Imdadul Islam*
}

\begin{abstract}
WiMAX is intended for fourth generation wireless mobile communications where a group of users are provided with a connection and a fixed length queue. In present literature traffic of such network is analyzed based on the generator matrix of the Markov Arrival Process (MAP). In this paper a simple analytical technique of the two dimensional Markov chain is used to obtain the trajectory of the congestion of the network as a function of a traffic parameter. Finally, a two state phase dependent arrival process is considered to evaluate probability states. The entire analysis is kept independent of modulation and coding schemes.
\end{abstract}

Keywords - WiMAX, Markov Arrival Process, Markov Modulated Poisson Process, Two dimensional Markov Chain

\section{INTRODUCTION}

The high speed data transfer requirement through mobile devices results in the advent of $4 \mathrm{G}$ mobile technology. Since the mobile WiMAX (IEEE 802.16e) already has the key building blocks required by 4G (incorporation of OFDMA, flat-all IP- network, fixed or mobile operation, MIMO, hybrid ARQ, multi-megabit speeds delivered to users, etc.), it is considered to be $4 \mathrm{G}$ mobile cellular technology. Originally, the IEEE 802.16 standard was developed for fixed wireless to allow homes and businesses to link with the worldwide core network since it was predicted that the IEEE 802.16 standard would be a better option for multipurpose connections, compared to fiber, cable, or digital subscriber line (DSL) links, because wireless systems are less costly to deploy over wide geographic areas [1].

Although WiMAX promises high speed wireless data transmission, the performance of the wireless networks will be highly dependent on how well radio resource management supports quality-of-service (QoS) requirements since, unlike wired networks, wireless networks are highly dependent on communications channels and radio channels are dynamic, correlated, unreliable, and are heavily affected by fading. Therefore, several cross-layer issues between the medium access control (MAC) layer and the physical (PHY) layer should be optimally resolved on the radio resource management side of the WiMAX systems [2]. Let us consider a single cell with a base station and multiple subscriber stations in a WiMAX network shown in Fig.1. Each subscriber station can serve multiple connections.

\footnotetext{
Manuscript received May 16, 2011; first revision August 26; accepted October 27, 2011.

Corresponding Author: Tonmoy Saha

* Dept. of Computer Science \& Engineering, Jahangirnagar University, Savar, Dhaka-1342, Bangladesh (tonmoy sh102@yahoo.com,sufean@gmail.com,dipu200002@yahoo.com,imdad@juniv.edu)
} 
The number of ongoing connections through a subscriber station is limited by applying admission control to each subscriber station. Traffic from all uplink connections are aggregated into a single queue at each subscriber station. Since the size of the queue is finite some packets will be dropped upon their arrival if the queue is full. The head of the line packet(s) are retrieved and transmitted to the base station by the OFDMA transmitter at the subscriber station. A different number of sub channels may be allocated to different subscriber stations by the base station; for example, the subscriber stations can be prioritized. A subscriber station having higher priority can be allocated more sub channels than a subscriber station having lower priority [3].

The Markov arrival process (MAP) is the most convenient technique for analyzing the traffic model of Fig. 1. Among the different types of MAPs, two states of the Markov Modulated Poisson Process (MMPP) model have been chosen for this paper. The MMPP is a double stochastic Poisson process where arrival rate of any traffic depends on its probability state, which forms a continuous time Markov chain. In a continuous time Markov chain, the sojourn time/life time in any state $i$ is exponentially distributed with parameter $\lambda_{i}$. At the end of sojourn time in state $i$, a transition takes place to another state or to the same state. The transition may or may not correspond to an arrival. Let us consider the simple case of a two state MMPP system where the arrival rate $\lambda_{i} ; i=1,2$ appears alternately with exponentially distributed life time $r_{i}^{-1} ; i=1,2$ shown in Fig. 2 (a) and (b) where the transition between level-1 and 2 occurs without any arrival. The 2 state MMPP is characterized by $(\mathbf{Q}, \mathbf{D})$ where $\mathbf{Q}$ is the infinitesimal generator and $\mathbf{D}$ is a $2 \times 2$ arrival matrix. The generator matrix $\mathbf{Q}$ is expressed as the sum of another two matrices $\mathbf{C}$ and $\mathbf{D}$. All the off-diagonal elements of $\boldsymbol{C}$ and all the elements of $\mathbf{D}$ are nonnegative but the diagonal elements of $\mathbf{C}$ are negative like [4],

$$
\mathrm{C}=\left[\begin{array}{cc}
-\left(r_{1}+\lambda_{1}\right) & r_{1} \\
r_{2} & -\left(r_{2}+\lambda_{2}\right)
\end{array}\right], \mathrm{D}=\left[\begin{array}{ll}
\lambda_{1} & 0 \\
0 & \lambda_{2}
\end{array}\right] \text { and } \mathrm{Q}=\mathrm{C}+\mathrm{D}=\left[\begin{array}{cc}
-r_{1} & r_{1} \\
r_{2} & -r_{2}
\end{array}\right]
$$

Now-a-days wide applications of the MMPP traffic model are found in current literature. In

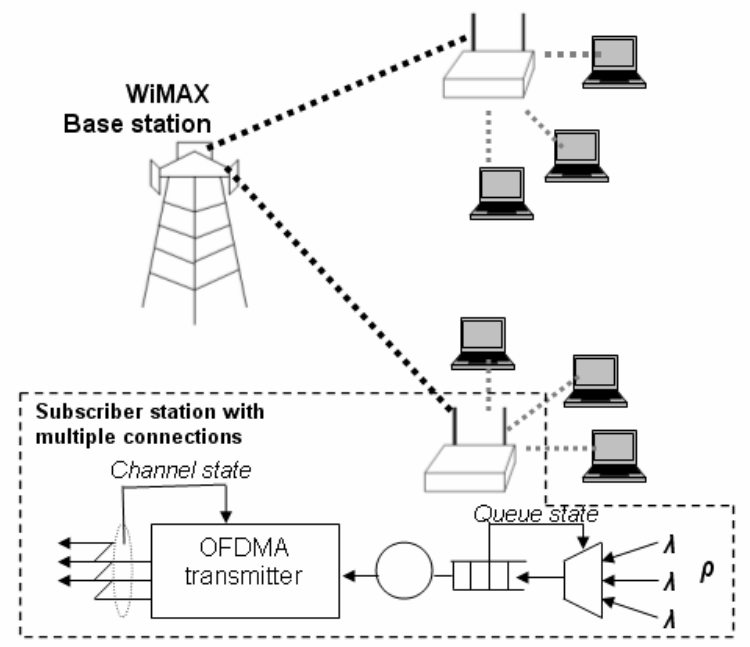

Fig. 1. WiMAX traffic model 


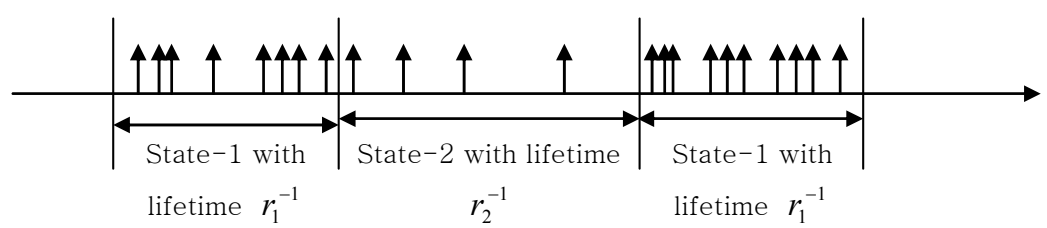

(a) Packet arrival and sojourn time

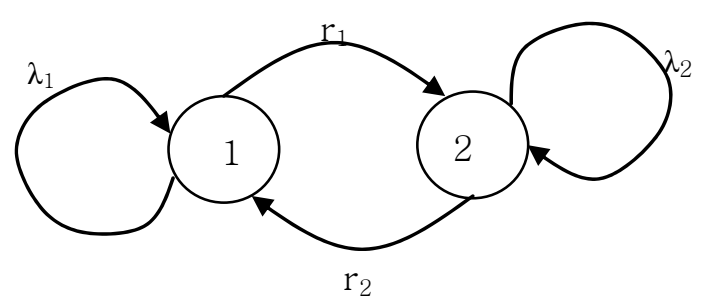

(b) State transition chain

Fig. 2. The two states of MMPP

[5] the authors deal with the waiting time of MMPP(2) traffic, which is applicable to real-time ATM traffic. The paper first shows the evaluation technique of waiting time based on a generator matrix and Laplace transform of [6]. The authors finally suggest an approximate technique following the two-term exponential function. The results of both techniques are found very close. The extension of analysis of [5] is given in [7] where the authors proposed a new traffic model called "suppressed ATM traffic" using MMPP(2). The mean and autocorrelation of cell interarrival times, and the histogram of the number of arrivals are measured. The authors also extends the MMPP(2) to a MAP(3) by adding a new state with an inter-state transition accompanied by an arrival. The analysis of the paper shows that both the proposed MMPP(2) and MAP(3) show a very good estimation of the cell loss ratio for usual superposition of voice and/or VBR video sources. The performance of transmission for Telemedicine using an ATM network is determined in [8]. The paper shows the profile of the probability of overflow for a queue against the buffer size based on the MMPP model. The packet loss rate of MMPP/M /1/ K traffic for a variable length packet is determined in [9]. In [10] the performance of VoIP traffic is analyzed for a cognitive radio system using the two state MMPP model. The average queue length and packet dropping probability of VoIP traffic is analyzed based on statistical multiplexing of two-state MMPP in [11]. The authors of [12] claimed an approximate method to evaluate the performance of voice and MMPP video traffic. In [13] constant and variable bit rate traffic is modeled under an ATM network and throughputs for videoconferencing are calculated.

The rest of the paper is organized as follows: Section 2 deals with the traffic model of a WLAN. Section 3 deals with the steady state probability states of two dimensional Markov chain of WiMAX traffic and Section 4 provides a traffic model for the phase dependent arrival process for WiMAX traffic. Section 5 depicts the results of the theoretical analysis and finally Section 6 concludes the entire analysis. 


\section{TRAFFIC MODEL OF THE WLAN}

The objective of this section is to derive the steady probability state of IEEE $802.11, \mathrm{M} / \mathrm{G} / 1 / \mathrm{k}$, $\mathrm{M} / \mathrm{M} / \mathrm{n}$ in generalized form to observe their profile together. Let us first consider the case of traffic of IEEE 802.11. In [14] authors deal with the IEEE 802.11 MAC protocol of WLAN to estimate the traffic parameters using three probability states $P_{i}$ (probability of idle state) $P_{s}$ (probability of successful transmission) and $P_{c}$ (the probability of collision). In [15] authors determined the throughput of IEEE 802.11 MAC traffic, based on the MMPP/G/1/m queuing model. A discrete time Markov chain is also proposed to determine probability states. The probability states are evaluated as:

$$
x_{0}=\gamma^{T}\left\{\theta_{0}(\mathbf{\Lambda}-\mathbf{Q})^{-1}\right\} E\left[T_{B}\right]^{-1}
$$

$n=1,2, \ldots \ldots \ldots, m-1$

$$
x_{n}=\gamma^{T}\left\{\theta_{n}+\sum_{k=0}^{n-1} \theta_{k} \mathbf{U}^{n-1-k}(\mathbf{U}-\mathbf{I})\right\}(\mathbf{\Lambda}-\mathbf{Q})^{-1} E\left[T_{B}\right]^{-1}
$$

and

$$
x_{m}=\theta-\sum_{n=0}^{m-1} x_{n}
$$

Where the matrices $\mathbf{D}$ and $\mathbf{Q}$ are given in Section $1, \mathbf{U}=(\boldsymbol{\Lambda}-\mathbf{Q})^{-\mathbf{1}} \boldsymbol{\Lambda}$, $\gamma=\frac{E\left[T_{B}\right]}{E\left[T_{B}\right]+\theta_{0}(\mathbf{\Lambda}-\mathbf{Q})^{-\mathbf{1}} \mathbf{e}}, \mathrm{E}\left[\mathrm{T}_{\mathrm{B}}\right]$ is the mean values of length of packets, $\theta$ is the steady-state probability vector. Equations (1) to (3) and the corresponding parameters are given with explanations in [15]. Similar analysis is applied in [16] for VoIP services in the IEEE 802.16e network.

Let us now consider the case of $\mathrm{M} / \mathrm{G} / 1 / \mathrm{m}$ traffic model. Let $\Pi_{j}^{*}$ is the probability that $j$ calls exist just after departure of a call in $\mathrm{M} / \mathrm{G} / 1$ system and $p_{j}$ is the probability that $j$ calls arrive during the service time.

Therefore according to [17-18]:

$$
\begin{aligned}
& \Pi_{j}^{*}=p_{j} \Pi_{0}^{*}+p_{j} \Pi_{1}^{*}+p_{j-1} \Pi_{2}^{*}+p_{j-2} \Pi_{3}^{*}+\ldots \ldots \\
& \Pi_{j}^{*}=p_{j} \Pi_{0}^{*}+\sum_{k=1}^{j+1} p_{j-k+1} \Pi_{k}^{*} ; j=0,1,2, \ldots
\end{aligned}
$$

For a finite queuing system, for example $\mathrm{M} / \mathrm{G} / 1 / \mathrm{m}, \quad \Pi_{j}^{*}=0$ for $j \geq m+1$. Let us derive a recurrence formula from (4). 


$$
\begin{aligned}
& \Pi_{j}^{*}=p_{j} \Pi_{0}^{*}+p_{j} \Pi_{1}^{*}+p_{j-1} \Pi_{2}^{*}+p_{j-2} \Pi_{3}^{*}+\ldots \quad \ldots \quad \ldots+p_{j-k+1} \Pi_{k}^{*}+\ldots \quad \ldots \quad \ldots+p_{0} \Pi_{j+1}^{*} \\
& \Rightarrow p_{0} \Pi_{j+1}^{*}=\Pi_{j}^{*}-\left(p_{j} \Pi_{0}^{*}+p_{j} \Pi_{1}^{*}+p_{j-1} \Pi_{2}^{*}+p_{j-2} \Pi_{3}^{*}+\ldots \quad \ldots \quad \ldots+p_{j-k+1} \Pi_{k}^{*}+\ldots \quad \ldots \quad \ldots+p_{1} \Pi_{j}^{*}\right) \\
& \Rightarrow p_{0} \Pi_{j+1}^{*}=\Pi_{j}^{*}-p_{j} \Pi_{0}^{*}-\sum_{k=1}^{j} p_{j-k+1} \Pi_{k}^{*} \\
& \therefore \Pi_{j+1}^{*}=\left(\Pi_{j}^{*}-p_{j} \Pi_{0}^{*}-\sum_{k=1}^{j} p_{j-k+1} \Pi_{k}^{*}\right) p_{0}^{-1}
\end{aligned}
$$

The detail derivation of (4) and (5) is given in appendix A.

$$
\text { Here } j=0,1,2,3, \ldots, m-1 \text { and } p_{j}=\frac{a^{j}}{j !} e^{-a}
$$

; where ' $a$ ' is the offered traffic intensity.

Taking, $C_{j}=\frac{\Pi_{j}^{*}}{\Pi_{0}^{*}}$ we get:

$$
C_{j+1}=\left(C_{j}-p_{j}-\sum_{k=1}^{j} p_{j-k+1} C_{k}\right) p_{0}^{-1} ; j=0,1,2, \ldots, m-1
$$

Let, $C=\sum_{j=0}^{m} C_{j}$ with $C_{0}=1$

Now combining above equations,

$$
\Pi_{j}=\Pi_{j}^{*}=P_{j}=\frac{C_{j}}{1+a C}
$$

The steady probability state of $\mathrm{M} / \mathrm{M} / \mathrm{n}$ is simply the Erlang's traffic given as, $P_{j}=\frac{\left(\lambda t_{h}\right)^{j} / j}{\sum_{k=0}^{n}\left(\lambda t_{h}\right)^{k} / k !}$, where $\lambda$ and $t_{h}$ are the average call arrival rate and holding time.

Fig. 3 compares the probability states of Markovian and Non-Markovian traffic models. It is visualized that the steady state probability states of IEEE 802.11/IEEE 802.16 of [14-16], $\mathrm{M} / \mathrm{G} / 1 / \mathrm{m}$ and $\mathrm{M} / \mathrm{M} / \mathrm{n}$ are very closed. Therefore for the two-dimensional traffic model of IEEE 802.16/ WiMAX we can use the Markovian traffic model. Although Fig. 1 shows the profile of $\mathrm{M} / \mathrm{E} 2 / 1 / \mathrm{k}$ and $\mathrm{M} / \mathrm{H} 2 / 1 / \mathrm{k}$, their derivation is not included here since their profile does not match with IEEE 802.11 traffic. The detailed analyses of them are given in [17]. 


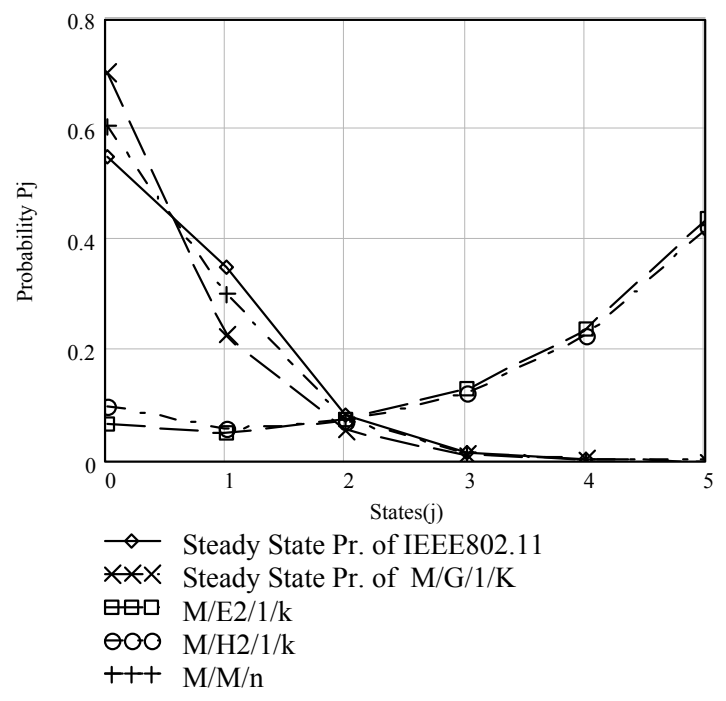

Fig. 3. Comparison of probability states

\section{Steady state Markov Chain Model}

Let us now represent the probability states of the traffic by a two dimensional Markov chain where any probability state $(\mathrm{C}, \mathrm{X})$ indicates $\mathrm{C}$ connections and $\mathrm{X}$ packets in queue for a group of users. Here $\rho$ is the connection arrival rate, $\lambda$ is the packet arrival rate, $\mu_{1}$ is the connection termination rate, and $\mu_{2}$ is the packet termination rate. Instead of incorporating the $\mathbf{Q}, \mathbf{P}$, and $\mathbf{V}$ matrix of [19], here only a cut equation technique is applied to solve the Markov chain and the system is kept independent of a modulation scheme.

Applying the cut equation on the $y^{\text {th }}$ column of Fig. 4 we get in generalized form [20] as:

$$
P_{x y}=P_{0 y}\left(\frac{\rho}{\mu_{1}}\right)^{x} \frac{1}{x !} .
$$

Similarly for $\mathrm{x}^{\text {th }}$ row of Fig.4:

$$
P_{x y}=P_{x 0}\left(\frac{\lambda x}{\mu_{2}}\right)^{y} \frac{1}{y !} .
$$

Now putting $y=0$ in equation (9) we get:

$$
P_{x 0}=P_{00}\left(\frac{\rho}{\mu_{1}}\right)^{x} \frac{1}{x !} .
$$




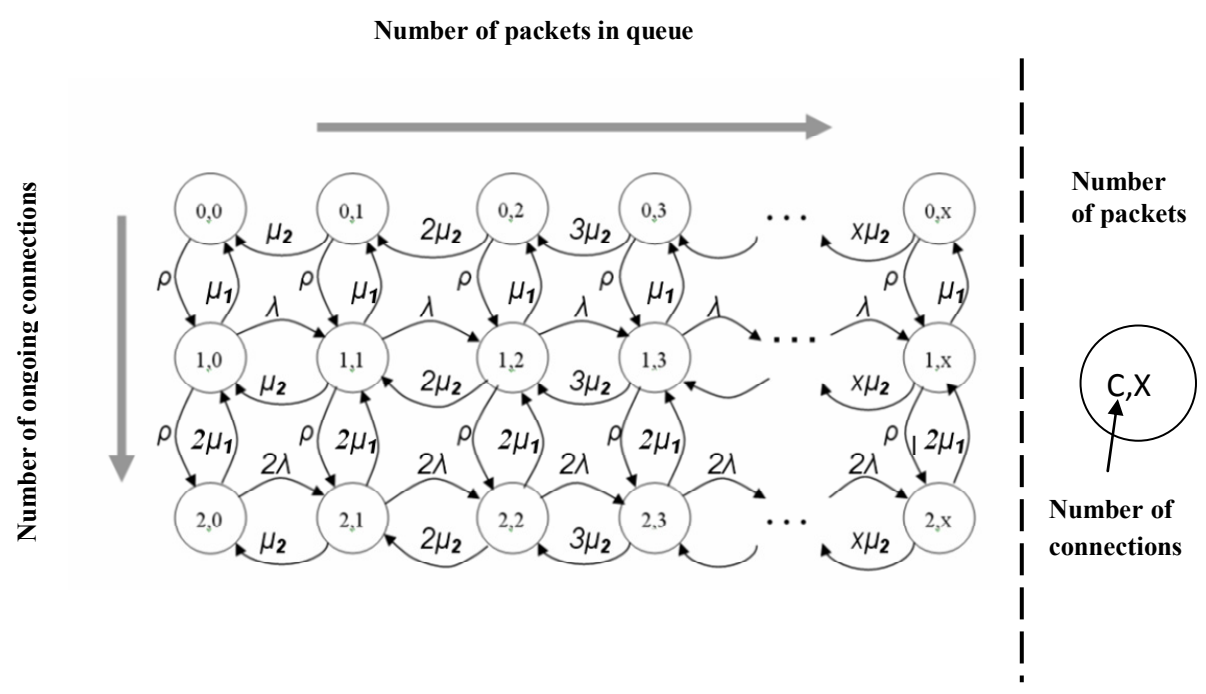

Fig. 4. Two Dimensional Markov Chain of WiMAX traffic

From (10) and (11):

$$
P_{x y}=P_{00}\left(\frac{\rho}{\mu_{1}}\right)^{x} \frac{1}{x !}\left(\frac{\lambda x}{\mu_{2}}\right)^{y} \frac{1}{y !}
$$

For an entire sampling space:

$\sum_{x=0}^{m} \sum_{y=0}^{n} P_{x y}=1$; where $\mathrm{m}$ and $\mathrm{n}$ are the maximum possible number of connections and packets for a link.

$$
\begin{gathered}
\Rightarrow P_{00} \sum_{x=0}^{m} \sum_{y=0}^{n}\left(\frac{\rho}{\mu_{1}}\right)^{x} \frac{1}{x !}\left(\frac{\lambda x}{\mu_{2}}\right)^{y} \frac{1}{y !}=1 \\
\therefore P_{00}=\frac{1}{\sum_{x=0}^{m} \sum_{y=0}^{n}\left(\frac{\rho}{\mu_{1}}\right)^{x} \frac{1}{x !}\left(\frac{\lambda x}{\mu_{2}}\right)^{y} \frac{1}{y !}}
\end{gathered}
$$

Putting $P_{00}$ in (12) we get, 


$$
P_{x y}=\frac{\left(\frac{\rho}{\mu_{1}}\right)^{x} \frac{1}{x !}\left(\frac{\lambda x}{\mu_{2}}\right)^{y} \frac{1}{y !}}{\sum_{x=0}^{m} \sum_{y=0}^{n}\left(\frac{\rho}{\mu_{1}}\right)^{x} \frac{1}{x !}\left(\frac{\lambda x}{\mu_{2}}\right)^{y} \frac{1}{y !}}
$$

Derivation of equations (9) to (12) is given in appendix B.

Now the sum of the complete occupied states that will provide the congestion probability can be expressed as:

$$
B=\sum_{i=0}^{M} P_{i, M-i} ; \text { where } \mathrm{M} \text { is the maximum possible value of } \mathrm{m}+\mathrm{m} \text { i.e. } \mathrm{M} \leq \mathrm{x}+\mathrm{y}
$$

\section{WIMAX USING MMPP}

WiMAX traffic can be analyzed based on two levels of the Markov Modulated Poisson Process (MMPP) model like Fig. 5 where the first column of the Markov chain indicates the underload state and that of the second column indicates the overload state of the network. For a particular column, the sizes of packets are fixed but numbers of connections are variable like

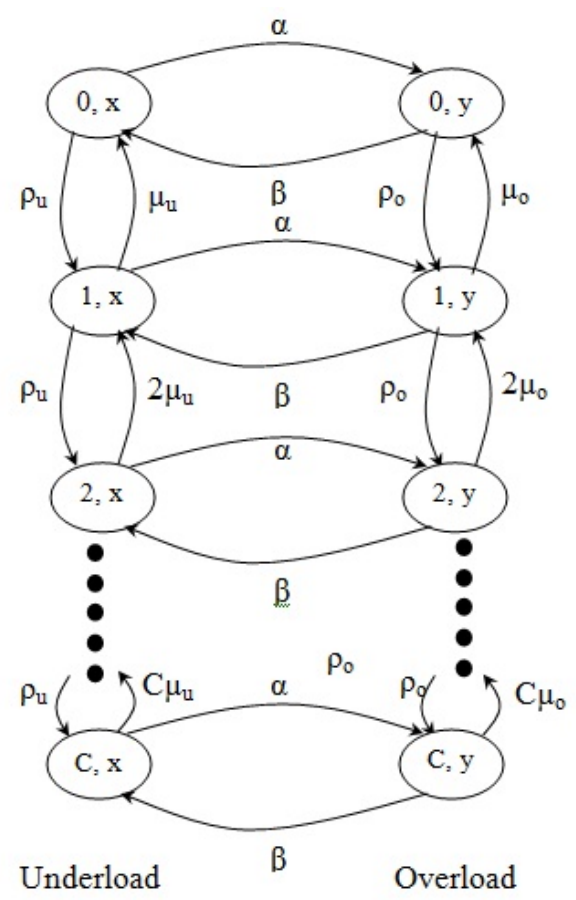

Fig. 5. State diagram between the underload and overload states 
[21]. The traffic parameters are,

$\alpha=$ Birth rate while the process transits in a state.

$\beta=$ Transition rate of the process from one state to another.

$\rho_{\mathrm{u}}=$ Arrival rate for the underload state.

$\rho_{0}=$ Arrival rate for the overload state.

$\mu_{\mathrm{u}}=$ Service rate for the underload state.

$\mu_{0}=$ Service rate for the underload state.

Let the Eigen values of the generator matrix $\boldsymbol{Q}$ of dimension of $\mathrm{i} \times \mathrm{i}$ be $\sigma_{l}, \sigma_{2}, \ldots \sigma_{i}$. Now the diagonal matrix [4]:

$$
\mathbf{D}(t)=\left[\begin{array}{cccc}
e^{\sigma_{1} \mathrm{t}} & 0 & \cdots & 0 \\
0 & e^{\sigma_{2} \mathrm{t}} & \cdots & 0 \\
\vdots & 0 & \ddots & \vdots \\
0 & 0 & \cdots & e^{\sigma_{\mathrm{i}} \mathrm{t}}
\end{array}\right]
$$

Let $\boldsymbol{M}$ be the service rate matrix of size i $\times$; where each column of $\boldsymbol{M}$ is the corresponding eigen vector of $\boldsymbol{Q}$.

Now the probability states as a function of time are:

$$
P(t)=\boldsymbol{\pi} \mathbf{M D}(t) \mathbf{M}^{-1}
$$

Where, $\boldsymbol{\pi}$ is the initial probability vector.

From Fig. 5 the balance equation for the system with $\mathrm{n} \geq 1$ and $0<\mathrm{i}<\mathrm{K}$ is expressed as:

$$
\left(\rho_{i}+\mu_{i}+\alpha_{i}+\beta_{i}\right) P_{n i}=\rho_{i} P_{n-1, i}+\alpha_{i-1} P_{n, i-1}+\beta_{i+1} P_{n, i+1}+\mu_{i} P_{n+1, i}
$$

After solving the balance equation we found that:

$$
G(z) \mathbf{A}(z)=(1-z) P_{0} \mathbf{M}
$$

where: $\mathbf{A}(z)=\Lambda z^{2}-(\mathbf{\Lambda}-\mathbf{Q}+\mathbf{M}) z+\mathbf{M}$

Roots of $\operatorname{det} \mathbf{A}(z)$ are grouped as: $z^{(0,1)}$ lies between 0 and $1, z^{(1, \infty)}$ lies between $1^{+}$and $\infty$ and $z=1$.

For $z=1$ :

$$
1=\left.\frac{1}{[\operatorname{det} A(z) /(1-z)]_{z=1}} P_{0} \operatorname{MAdj} A(z)\right|_{z=1} e
$$

Where $e$ is a column vector of $1 \mathrm{~s}$. 
For $z<1$ :

$$
0=\mathbf{P}_{0} \mathbf{M} \operatorname{Adj} \mathbf{A}(z) \mathbf{e}
$$

From (17) the probability generating function can be written as below:

$$
G(z)=\frac{1-z}{\operatorname{det} A(z)} P_{0} \operatorname{MAdjA}(z)
$$

Using equations (17) to (20), the probability states of Fig. 5 can be determined for any value of $n$.

\section{Example:}

Let the generator and diagonal matrices be:

$$
\mathbf{Q}=\left[\begin{array}{cc}
-\alpha & \alpha \\
\beta & -\beta
\end{array}\right]=\left[\begin{array}{cc}
-0.2 & 0.2 \\
0.3 & -0.3
\end{array}\right]
$$

The service and arrival rates (diagonal) matrices are:

$$
\mathbf{M}=\left[\begin{array}{cc}
\mu_{u} & 0 \\
0 & \mu_{0}
\end{array}\right]=\left[\begin{array}{ll}
1 & 0 \\
0 & 1
\end{array}\right] \text { and } \Lambda=\left[\begin{array}{cc}
\rho_{u} & 0 \\
0 & \rho_{0}
\end{array}\right]=\left[\begin{array}{cc}
0.6 & 0 \\
0 & 0.85
\end{array}\right]
$$

From the substitution of $\mathbf{Q}, \mathbf{M}$ and $\Lambda$ into (18) we get:

$$
A(z)=\left[\begin{array}{cc}
0.6 z^{2}-1.8 z+1 & 0.2 z \\
0.3 z & 0.85 z^{2}-2.15 z+1
\end{array}\right]
$$

Taking the adjoint of $A(z)$ and the determinate of $A(z)$ :

$$
\begin{aligned}
\operatorname{Adj} A(z) & =\left[\begin{array}{cc}
0.85 z^{2}-2.15 z+1 & -0.2 z \\
-0.3 z & 0.6 z^{2}-1.8 z+1
\end{array}\right] \\
\operatorname{det} A(z) & =0.510 z^{4}-2.820 z^{3}+5.260 z^{2}-3.95 z+1
\end{aligned}
$$

The roots of $\operatorname{det} A(z)$ are $z^{(0,1)}=0.5403, \quad z^{(1)}=1$ and $z^{(1, \infty)}=1.4035,2.5856$

Now, for $z^{(1)}=1$ :

$$
\frac{1}{-0.294} M\left[\begin{array}{ll}
-0.3 & -0.2 \\
-0.3 & -0.2
\end{array}\right]\left[\begin{array}{l}
1 \\
1
\end{array}\right]=\left[\begin{array}{l}
1.701 \\
1.701
\end{array}\right]
$$


For $z^{(0,1)}=0.5403$ :

$$
M\left[\begin{array}{cc}
0.086 & -0.108 \\
-0.162 & 0.203
\end{array}\right]\left[\begin{array}{l}
1 \\
1
\end{array}\right]=\left[\begin{array}{c}
-0.022 \\
0.041
\end{array}\right]
$$

Now from equation (11) and (12):

$$
\begin{gathered}
{\left[\begin{array}{ll}
0 & 1
\end{array}\right]=P_{0}\left[\begin{array}{cc}
-0.022 & 1.701 \\
0.041 & 1.701
\end{array}\right]} \\
\therefore P_{0}=\left[\begin{array}{ll}
0.383 & 0.205
\end{array}\right]
\end{gathered}
$$

The probability generating function:

$$
G(z)=\frac{1-z}{\operatorname{det} A(z)} P_{0} \mathbf{M} \operatorname{Adj} \mathbf{A}(z)
$$

Substituting the results into the above equation we get:

$$
G(z)=\left[\frac{0.383(1-z)\left(0.85 z^{2}-2.45 z+1.3\right)-0.065(1-z) z}{0.510 z^{4}-2.820 z^{3}+5.260 z^{2}-3.95 z+1} \quad \frac{-0.0766(1-z) z+0.205(1-z)\left(0.6 z^{2}-2.1 z+1.3\right)}{0.510 z^{4}-2.820 z^{3}+5.260 z^{2}-3.95 z+1}\right]
$$

After solving the above equation:

$$
G_{0}(z)=0.383+\frac{0.0847}{1-0.6972 z}
$$

and $G_{1}(z)=0.205+\frac{0.5609}{1-0.6972 z}$

Writing $G_{0}(z)$ and $G_{1}(z)$ in a matrix form:

$$
\left[G_{0}(z) \quad G_{1}(z)\right]=\left[\begin{array}{ll}
0.383 & 0.205
\end{array}\right]+\left[\begin{array}{ll}
0.0847 & 0.5609
\end{array}\right] \sum_{n=1}^{\infty}(0.6907)^{n} z^{n}
$$

Thus, for $\mathrm{n} \geq 1$ :

$$
\left[\begin{array}{ll}
P_{n 0} & P_{n 1}
\end{array}\right]=\left[\begin{array}{ll}
0.0847 & 0.5609
\end{array}\right](0.6907)^{n}
$$

Equation (22) provides the probability states of Fig. 5. 


\section{Results AND Discussion}

This section provides probability states and packet dropping probability against states of the network and arrival rate by taking other factors as parameters. For a fixed number of connections with a variable number of packet arrival, the probability states, $P_{y}$ are shown in Fig. 6 for two different number of connections $\mathrm{m}=5$ and $\mathrm{m}=8$. The profile of the probability states is neither linear nor like Poisson's probability distribution function since arrival rate $\lambda$ changes with the number of connections shown in Fig. 4. The state of connection, $P_{x}$ resembles $\mathrm{M} / \mathrm{M} / \mathrm{n}$ traffic (i.e. Poisson's probability density function visualized from Fig. 7 for two different queue lengths $[\mathrm{n}=15$ and $\mathrm{n}=10]$ ).

Packet dropping probability against connection arrival rate is plotted in Fig. 8 for three different queue lengths, where dropping probability increases with the increase in $\rho$ but decreases with the increase in queue length, supports the convention of teletraffic engineering. For $\rho \geq 0.65$ the packet dropping probability is less sensitive compared to the region of $\rho<0.65$.

Fig. 9 depicts the packet dropping probability against the packets arrival rate for three differ-

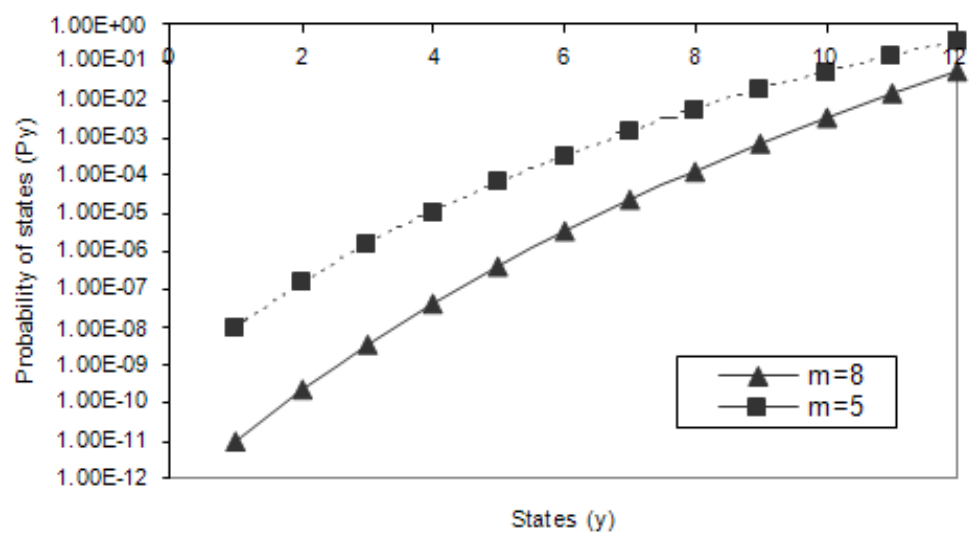

Fig. 6. Probability states of packets for a fixed connection $(\rho=1.5$ connection/unit time, $\mu 2=2$ connection/unit time, $\mu 1=5$ packet/unit time, $m=5, \lambda=30$ packets/unit time)

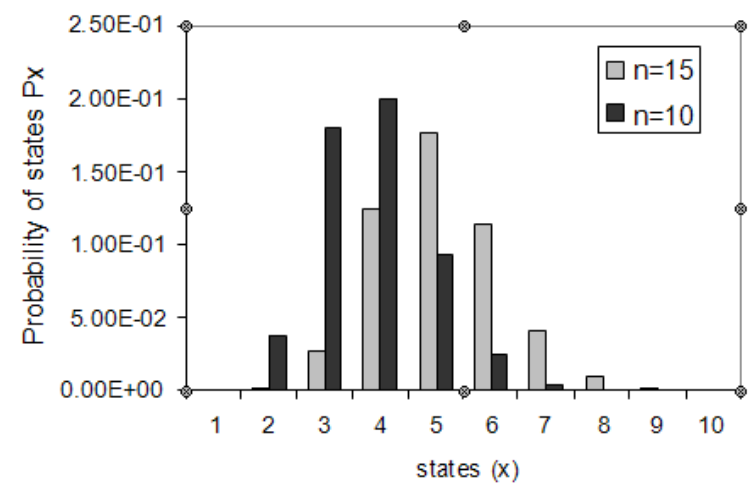

Fig. 7. Probability states of connection for a fixed queue length $\rho=0.5, \mu 2=2, \mu 1=5, n=10, \lambda=30$ 


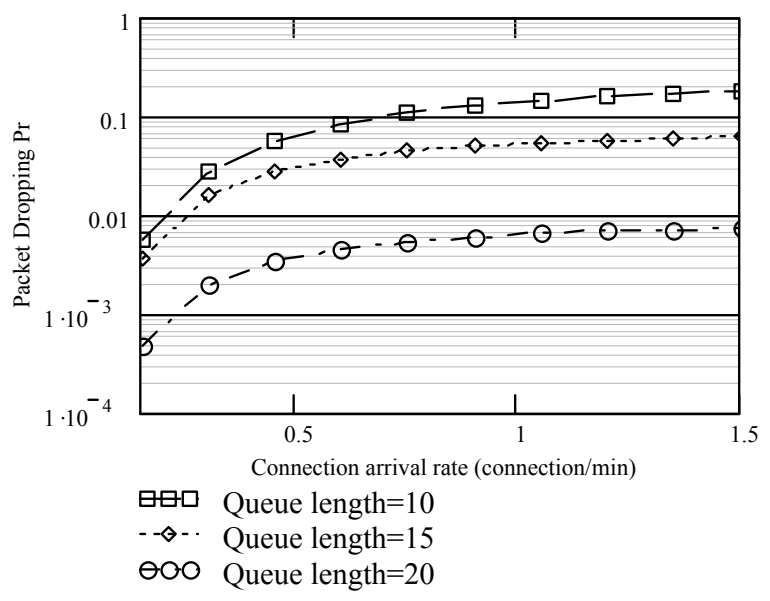

Fig. 8. Packet dropping probability against connection arrival rate taking the queue length as a parameter $(\mu 2=2, \mu 1=5, m=4, \lambda=15)$

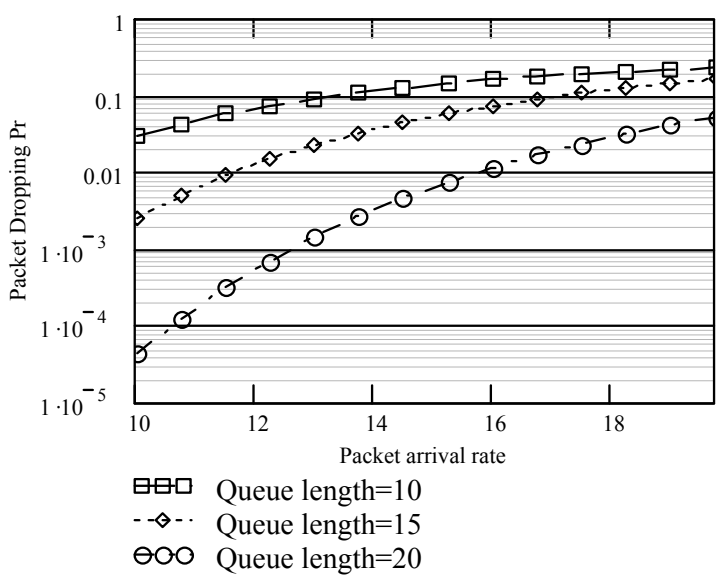

Fig. 9. Packet dropping probability against packet arrival rate taking the queue length as a parameter $(\rho=1, \mu 2=2, \mu 1=5, m=4, \lambda=30)$

ent queue lengths. For $\lambda \geq 14$ the dropping probability seems to be flat and at the same time, the impact of the queue length is also less sensitive in dropping probability.

Fig. 10 shows the profile of the probability states of MMPP as a function of the time taking initial probability vector $\Pi=\left[\begin{array}{ll}1 & 0\end{array}\right]$. Initially, the trajectory of both curves is exponential but after some sojourn time they become flat. The probability of staying in an underload condition is greater than that of being in an overload state for ensuring that the network operates with reasonable QoS. Finally, the probability of overload states $\mathrm{P}(\mathrm{n}, \mathrm{x})$ and underload states $\mathrm{P}(\mathrm{n}, \mathrm{y})$ for 
different values of $\mathrm{n}$ of Fig. 5 is shown in Fig. 11.

It is visualized that the profile of probability states of the underload condition follows the negative exponential distribution and the overload state follows the Poisson distribution.

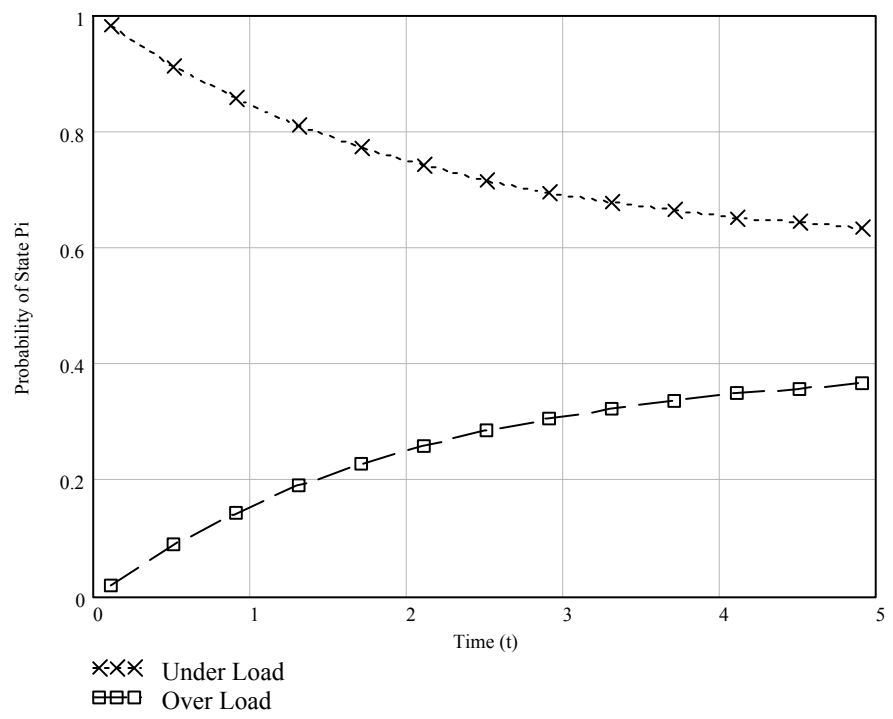

Fig. 10. Probability states as a function of time

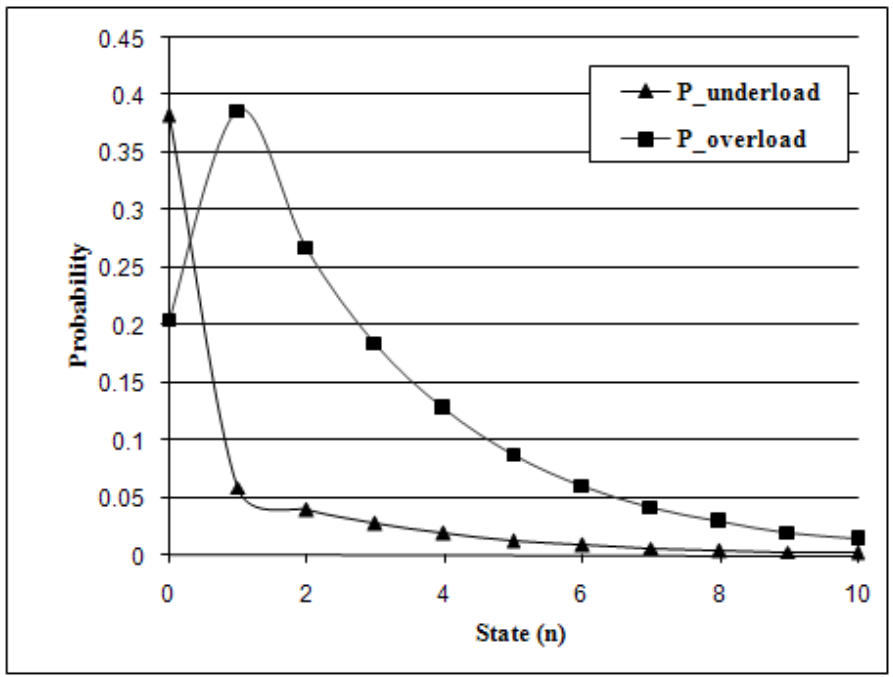

Fig. 11. Probability states of the phase dependent arrival process 


\section{CONCLUSION}

The paper deals with the traffic performance of a WiMAX network based on both the 2D Markov chain and the two levels of the MMPP model. Based on our analysis, network planners should optimize the throughput/carried traffic of a network with the proper number of connection and queue lengths. In this paper we consider that both inter-arrival time and service time follows Poisson's probability density function (pdf) for simplicity of analysis. In case of packet traffic of for $3 \mathrm{G}$ wireless communications, the sojourn time of the probability states follows the general or deterministic probability distribution functions. Therefore an M/G/n/m or M/D/n/m traffic model can be considered as an extension of the paper to present the traffic of IEEE 802.16, including adaptive modulation and channel coding scheme.

\section{REFERENCES}

[1] D. Rouffet, S. Kerboeuf, L. Cai, V. Capdevielle, “4G MOBILE, “ Alcatel Telecommunications Review-2nd Quarter, 2005.

[2] Kwang-Cheng Chen, "Introduction to Mobile WiMAX," NTUEE Mobile Communications, 2007.

[3] H. Fattah and C. Leung, "An Overview Scheduling Algorithms in Wireless Multimedia Networks," IEEE Wireless Communications, October 2002, pp.76-83.

[4] Md. Imdadul Islam, "Modeling of Voice Data Integrated Traffic for 3G Mobile Cellular Networks," Ph.D. Thesis Dept. of CSE, Jahangirnagar University, Dhaka, August 2009.

[5] Sang H. Kang, Dan K. Sung and Bong D. Choi, “An Empirical Real-Time Approximation of Waiting Time Distribution in MMPP(2)/D/1," IEEE Communication Letters, Vol.2, No.1, January 1998, pp.17-19.

[6] D. M. Lucantoni, "New results on the single server queue with a batch Markovian arrival process," Commun. Statist.-Stochastic Models, Vol.7, No.1, 1991, pp.1-46.

[7] Sang H. Kang, Yong Han Kim, Dan K. Sung and Bong D. Choi, "An application of Markovian Arrival Process (MAP) to modeling superposed ATM cell stream," IEEE Transaction on Communications, Vol.50, No.4, April 2002, pp.633-642.

[8] J. P. Dubois, and H. M. Chiu, "High Speed Video Transmission for Telemedicine using ATM Technology," World Academy of Science, Engineering and Technology 12, 2005, pp.103-107.

[9] L. P Raj Kumar, K. Sampath Kumar, D. Mallikarjuna Reddy, Malla Reddy Perati, "Analytical Model for Performance Study of the Switch under Self-Similar Variable Length Packet Traffic," Proceedings of the World Congress on Engineering and Computer Science 2010 Vol I WCECS 2010, October 20-22, 2010, San Francisco, USA.

[10] Howon Lee and Dong-Ho Cho, "VoIP Capacity Analysis in Cognitive Radio System," IEEE Communications Letters, Vol.13, No.6, June 2009, pp.339-395.

[11] Jae-Woo So, "Performance Analysis of VoIP Services in the IEEE 802.16e OFDMA System With Inband Signaling," IEEE TRANSACTIONS ON VEHICULAR TECHNOLOGY, Vol.57, No.3, May 2008, pp.1876-1986. 
[12] T.C. Wang, J. W. mark, K.C. Chua, "Delay performance of voice and MMPP video traffic in cellular wireless ATM network," IEE Proc. Commun., Vol.148, No.5, Oct' 2001, pp.302-309.

[13] Kyoko Yamori and Haruo Akimaru, "Optimum Design of Videoconferencing Systems over ATM Networks," Electronics and Communications in Japan, Part 1, Vol.85, No.6, 2002, pp.33-40.

[14] Chuan Heng Foh, Moshe Zukerman and Juki Wirawan Tantra, “A Markovian Framework for Performance Evaluation of IEEE 802.11," IEEE Transactions on Wireless Communications, Vol.6, No.4, April 2007, pp.1275-1285.

[15] C. Geun Park, D. Hwan Han, S. Joon Ahn, "Performance analysis of MAC layer protocols in the IEEE 802.11 wireless LAN," Telecommun Syst (2006) 33:233-253.

[16] Jae-Woo So, "Performance Analysis of VoIP Services in the IEEE 802.16e OFDMA System With Inband Signaling," IEEE Transactions on Vehicular Technology, Vol.57, No.3, May 2008, pp.1876-1886.

[17] Haruo Akimaru and Konosuke Kawashima, Teletraffic Theory and Applications, SpringerVerlag, Berlin, 1993.

[18] Kyoko Yamori and Haruo Akimaru, "Optimum Design of Videoconferencing Systems over ATM Networks," Electronics and Communications in Japan, Part 1, Vol.85, No.6, 2002, pp.33-40.

[19] Dusit Niyato and Ekram Hossain, Connection Admission Control in OFDMA-Based WiMAX Networks: Performance Modeling and Analysis, WiMAX/MobileFi, Advanced Research and Technology, edited by Yiang Xiao, Aurebach Publications, Taylor and Francis group, 2008, pp.1-29.

[20] Md. Imdadul Islam and Liton Jude Rozario, "Telecommunications Traffic and Network Planning, "Christian Communication Centre Pratibeshi Prokashani, June 2008.

[21] John N. Daigle, Queueing Theory with Applications to Packet Tele Communication, Springer Science and Business Media, Inc, Boston, eBook ISBN: 0-387-22859-4, 2005. 


\section{APPENDIX-A}

\section{Non-Markovian arrival process}

Let, $\mathrm{X}$ as a random variable indicate the interval between the consecutive occurrence of two events, for example, time difference between the arrival of two calls (inter arrival time). If the distribution function of $X$ is $F(x)$, which is independently and identically distributed (i.i.d), then the process $\{X\}$ is called a renewal process. The time interval between an observation point or the instant to the instant of the next occurrence is also a random variable $X^{*}$, is called the residual (life) time or forward occurrence time visualized from Fig. A.1. The CDF of $X^{*}$ is expressed as:

$$
R(t)=\frac{1}{m} \int_{0}^{t}[1-F(\xi)] d \xi ; \text { where } m=E[x] .
$$

$\therefore$ The PDF of $X^{*}$ is,

$$
r(t)=\frac{1}{m}[1-F(t)]
$$

The Poisson process is a special case of a renewal process where $F(x)$ follows exponential distribution. The Laplace-Stieltjes transform (LST) of $F(x)$ is expressed as:

$$
f^{*}(\theta)=\int_{0}^{\infty} e^{-\theta x} d F(x) \equiv \int_{0}^{\infty} e^{-\theta x} f(x) d x
$$

Where, $f(x)=\frac{d F(x)}{d x}$ is the PDF of random variable $X$.

$\therefore$ The LST of $R(t)$,

$$
\begin{aligned}
r^{*}(\theta) & =\int_{0}^{\infty} e^{-\theta t} d R(t) \\
& =\int_{0}^{\infty} e^{-\theta t} r(t) d t ; \text { Where: } r(t)=\frac{d R(t)}{d t}
\end{aligned}
$$

Last Occurrence Observation point Next Occurrence

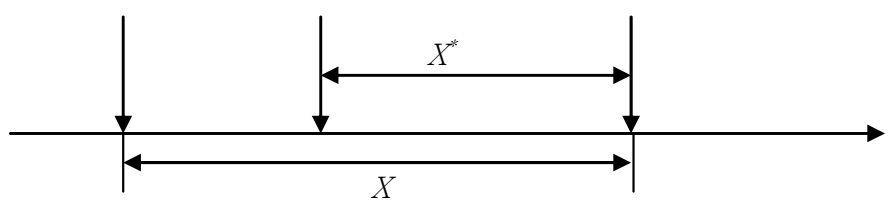

Fig. A.1. Renewal process 
From (A.2) and (A.4):

$$
\begin{aligned}
r^{*}(\theta) & =\int_{0}^{\infty} e^{-\theta t} \cdot \frac{1}{m}[1-F(t)] d t \\
& =\int_{0}^{\infty} \frac{e^{-\theta t}}{m} d t-\frac{1}{m} \int_{0}^{\infty} e^{-\theta t} F(t) d t \\
& =\left[-\frac{e^{-\theta t}}{m \theta}\right]_{0}^{\infty}-\frac{1}{m} I \\
& \therefore r^{*}(\theta)=\frac{1}{m \theta}-\frac{I}{m}
\end{aligned}
$$

Here: $I=\int_{0}^{\infty} e^{-\theta t} F(t) d t$

$$
\begin{aligned}
& =F(t) \int_{0}^{\infty} e^{-\theta t} d t-\int_{0}^{\infty}\left[\frac{d F(t)}{d t} \int e^{-\theta t} d t\right] d t \\
& =\left[\frac{F(t) e^{-\theta t}}{-\theta}\right]_{0}^{\infty}-\int_{0}^{\infty} \frac{F(t) e^{-\theta t}}{-\theta} d t \\
& =0+\frac{F(0)}{\theta}+\frac{1}{\theta} \int_{0}^{\infty} e^{-\theta t} F(t) d t \\
& =0+0+\frac{1}{\theta} f^{*}(\theta) ; \text { from }
\end{aligned}
$$

$$
\therefore I=\frac{1}{\theta} f *(\theta)
$$

From (A.5) and (A.6),

$$
\begin{aligned}
r *(\theta)= & \frac{1}{m \theta}-\frac{f^{*}(\theta)}{m \theta} \\
& \Rightarrow r *(\theta)=\frac{1-f^{*}(\theta)}{m \theta}
\end{aligned}
$$

The mean residual time:

$$
\begin{aligned}
R & =-\lim _{\theta \rightarrow 0} \frac{d r^{*}(\theta)}{d \theta} \\
& =-\lim _{\theta \rightarrow 0} \frac{d}{d \theta}\left[\frac{1-f^{*}(\theta)}{m \theta}\right]
\end{aligned}
$$




$$
\begin{aligned}
& =-\frac{1}{m} \lim _{\theta \rightarrow 0}\left[\frac{\theta \frac{d}{d \theta}\left\{1-f^{*}(\theta)\right\}-\left\{1-f^{*}(\theta)\right\} \frac{d}{d \theta} \theta}{\theta^{2}}\right] \\
& =-\frac{1}{m} \lim _{\theta \rightarrow 0}\left[\frac{-\theta f^{\prime *}(\theta)-\left\{1-f^{*}(\theta)\right\}}{\theta^{2}}\right] \\
& =\frac{1}{m} \lim _{\theta \rightarrow 0}\left[\frac{\theta f^{\prime *}(\theta)+1-f^{*}(\theta)}{\theta^{2}}\right] \\
& =\frac{1}{m} \lim _{\theta \rightarrow 0}\left[\frac{1 . f^{\prime *}(\theta)+\theta f^{\prime \prime} *(\theta)+0-f^{\prime *}(\theta)}{2 \theta}\right] \\
& =\frac{1}{m} \lim _{\theta \rightarrow 0}\left[\frac{\theta f^{\prime \prime} *(\theta)}{2 \theta}\right] \quad \therefore R=\frac{1}{2 m} f^{\prime \prime *}(0)
\end{aligned}
$$

From moment formula:

$$
\begin{aligned}
E\left[x^{k}\right] & =(-1)^{k} \lim _{\theta \rightarrow 0} \frac{d k}{d \theta^{k}} f^{*}(\theta) \\
& =(-1)^{k} f^{*(k)}(0)
\end{aligned}
$$

For $k=2$,

$$
E\left[x^{2}\right]=f^{\prime \prime} *(0)
$$

From (A.8) and (A.9):

$$
R=\frac{E\left[x^{2}\right]}{2 m}
$$

We know that: $E\left[x^{2}\right]=\sigma^{2}+m^{2}$

Let us define the severed coefficient of variation (SCV) by:

$$
\begin{array}{r}
c^{2}=\frac{\sigma^{2}}{m^{2}} \\
\therefore R=\frac{\sigma^{2}+m^{2}}{2 m} \\
=\frac{\sigma^{2}}{2 m}+\frac{m^{2}}{2 m} \\
=\frac{m}{2} c^{2}+\frac{m}{2}
\end{array}
$$




$$
=\frac{m}{2}\left(1+c^{2}\right)
$$

Let us consider the $\mathrm{M} / \mathrm{G} / 1$ system with an average arrival rate and service time of $\lambda$ and $\mathrm{h}$ respectively. Therefore the offered load is:

$$
a=\lambda h=P\{\text { the server is busy }\}
$$

Let $T_{1}$ in Fig. A.2 be the instant of termination of a test call and $L$ be the average number of calls waiting in queue at that instant. The instant $T_{2}$ of Fig. A.2 is an instant when the last call (among $\mathrm{L}$ calls) is in service and $T_{3}$ is the instant of its termination. The time between $T_{3}$ and $T_{2}$ is the mean residual time $R$ for the last call in service to be terminated. If the instant $T_{2}$ is ahead of the termination time of last call then $R=0$.

The mean waiting time: $W=a R+L h$. (A.11)

From the Little law: $L=\lambda W$. (A.12)

From (A.11) and (A.12),

$$
\begin{aligned}
W=a R+\lambda W h & \\
& \Rightarrow W(1-\lambda h)=a R \\
& \Rightarrow W(1-a)=a R \\
& \Rightarrow W=\frac{a R}{(1-a)}=\frac{a}{(1-a)} \frac{h}{2}\left(1+C^{2}\right) \\
& \because R=\frac{h}{2}\left(1+C^{2}\right)
\end{aligned}
$$

If $\mathrm{k}$ calls exist in a $\mathrm{M} / \mathrm{G} / 1$ system just after a call departure then the call at the front of the queue (FIFO case) enters service and the $j$ call arrives during its service time, then the number of calls present at its departure is: $k+j-1$.

Let $\Pi_{j}^{*}$ be the probability that $j$ calls exist just after departure of a call in the M/G/1 system and $p_{j}$ be the probability that $j$ calls arrive during the service time.

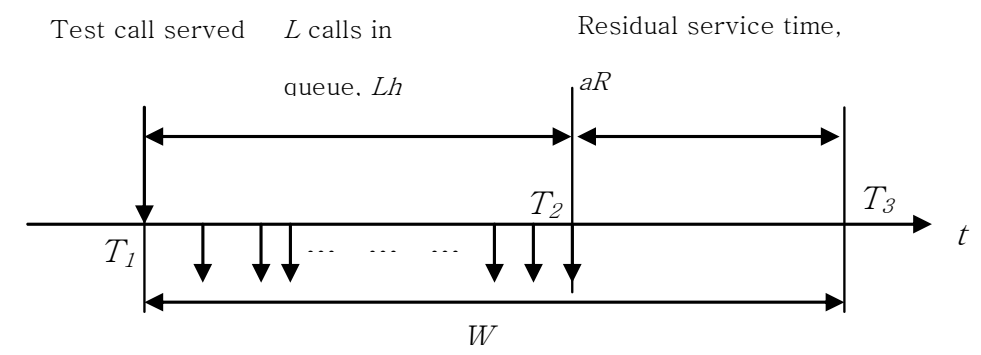

Fig. A.2. M/G/1 traffic model 
Therefore:

$$
\begin{gathered}
\Pi_{j}^{*}=p_{j} \Pi_{0}^{*}+p_{j} \Pi_{1}^{*}+p_{j-1} \Pi_{2}^{*}+p_{j-2} \Pi_{3}^{*}+\ldots \quad \ldots \quad \ldots+p_{j-k+1} \Pi_{k}^{*}+\ldots \quad \ldots \quad \ldots+p_{0} \Pi_{j+1}^{*} \\
\Pi_{j}^{*}=p_{j} \Pi_{0}^{*}+\sum_{k=1}^{j+1} p_{j-k+1} \Pi_{k}^{*} ; \mathrm{j}=0,1,2, \ldots
\end{gathered}
$$

For a finite queuing system, for example $\mathrm{M} / \mathrm{G} / 1(\mathrm{~m}), \Pi_{j}^{*}=0$ for $\mathrm{j} \geq \mathrm{m}+1$. Let us derive a recurrence formula from (A.14).

$$
\begin{gathered}
\Pi_{j}^{*}=p_{j} \Pi_{0}^{*}+p_{j} \Pi_{1}^{*}+p_{j-1} \Pi_{2}^{*}+p_{j-2} \Pi_{3}^{*}+\ldots \ldots \ldots+p_{j-k+1} \Pi_{k}^{*}+\ldots \ldots \ldots+p_{0} \Pi_{j+1}^{*} \\
\Rightarrow p_{0} \Pi_{j+1}^{*}=\Pi_{j}^{*}-\left(p_{j} \Pi_{0}^{*}+p_{j} \Pi_{1}^{*}+p_{j-1} \Pi_{2}^{*}+p_{j-2} \Pi_{3}^{*}+\ldots \ldots \ldots+p_{j-k+1} \Pi_{k}^{*}+\ldots \ldots \ldots+p_{1} \Pi_{j}^{*}\right) \\
\Rightarrow p_{0} \Pi_{j+1}^{*}=\Pi_{j}^{*}-p_{j} \Pi_{0}^{*}-\sum_{k=1}^{j} p_{j-k+1} \Pi_{k}^{*} \\
\therefore \Pi_{j+1}^{*}=\left(\Pi_{j}^{*}-p_{j} \Pi_{0}^{*}-\sum_{k=1}^{j} p_{j-k+1} \Pi_{k}^{*}\right) p_{0}^{-1}
\end{gathered}
$$

Where $j=0,1,2,3, \ldots, m-1$ and $p_{j}=\frac{a^{j}}{j !} e^{-a}$ 


\section{APPENDIX B}

\section{2-D Traffic}

If a communication network is in statistical equilibrium, it can be modeled as a multidimensional state transition diagram. Here the 2-D Markov chain is considered for a network having two types of Poisson's arrival traffic $A_{1}$ and $A_{2}$ (the network has $n=\infty$ servers or channels) as the simplest model. In this case it is convenient to arrange states $x_{1}$ and $x_{2}$ along the $X$ and $Y$ direction. For a K-dimensional traffic case, a K-dimensional co-ordinate system may be used. The probability state $P_{x I x z}$ (i.e. the probability of arrival of $x_{1}$ and $x_{2}$ calls of traffic of type- 1 and type-2 respectively as shown in Fig. B.1).

Let us consider the $\mathrm{x}_{2}$ th column of Fig. B.2 (a) for cut equations.

Applying the cut equation between first and second node:

$$
\begin{gathered}
\lambda_{1} P_{0 x_{2}}=P_{1 x_{2}} \mu_{1} \\
\therefore P_{1 x_{2}}=\frac{\lambda_{1}}{\mu_{1}} P_{0 x_{2}}=A_{1} P_{0 x_{2}}
\end{gathered}
$$

Between the second and third node:

$$
\begin{gathered}
\lambda_{1} P_{1 x_{2}}=P_{2 x_{2}} 2 \mu_{1} \\
\therefore P_{2 x_{2}}=\frac{\lambda_{1}}{2 \mu_{2}} P_{1 x_{2}}=\frac{A^{2}{ }_{1}}{2 !}
\end{gathered}
$$

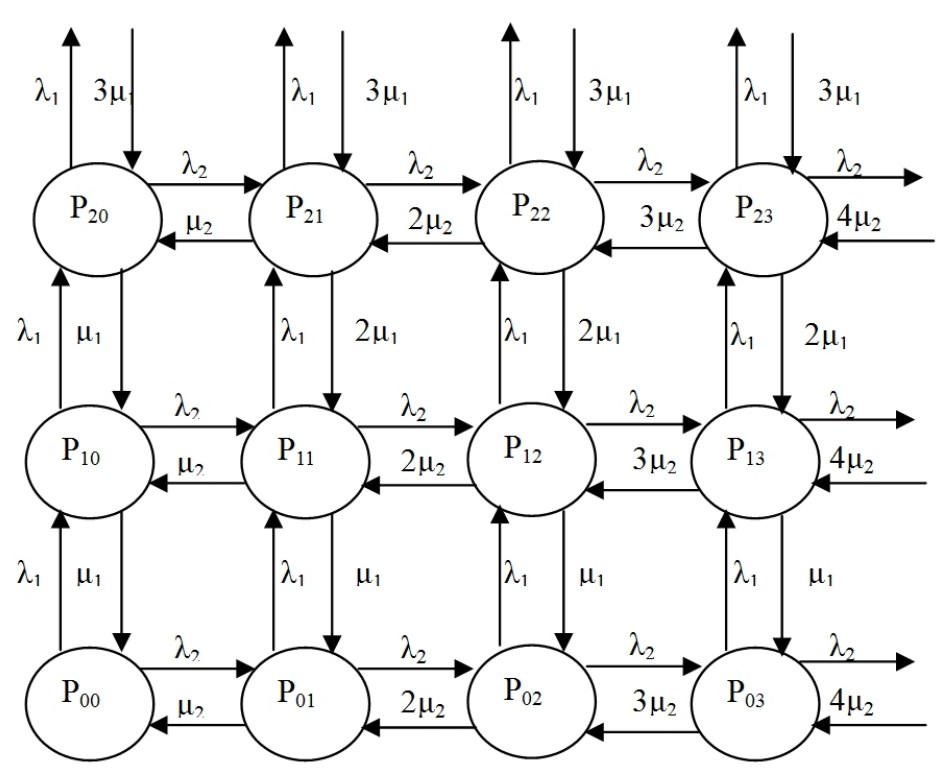

Fig. B.1. 2-D Markov chain of Poisson traffic 

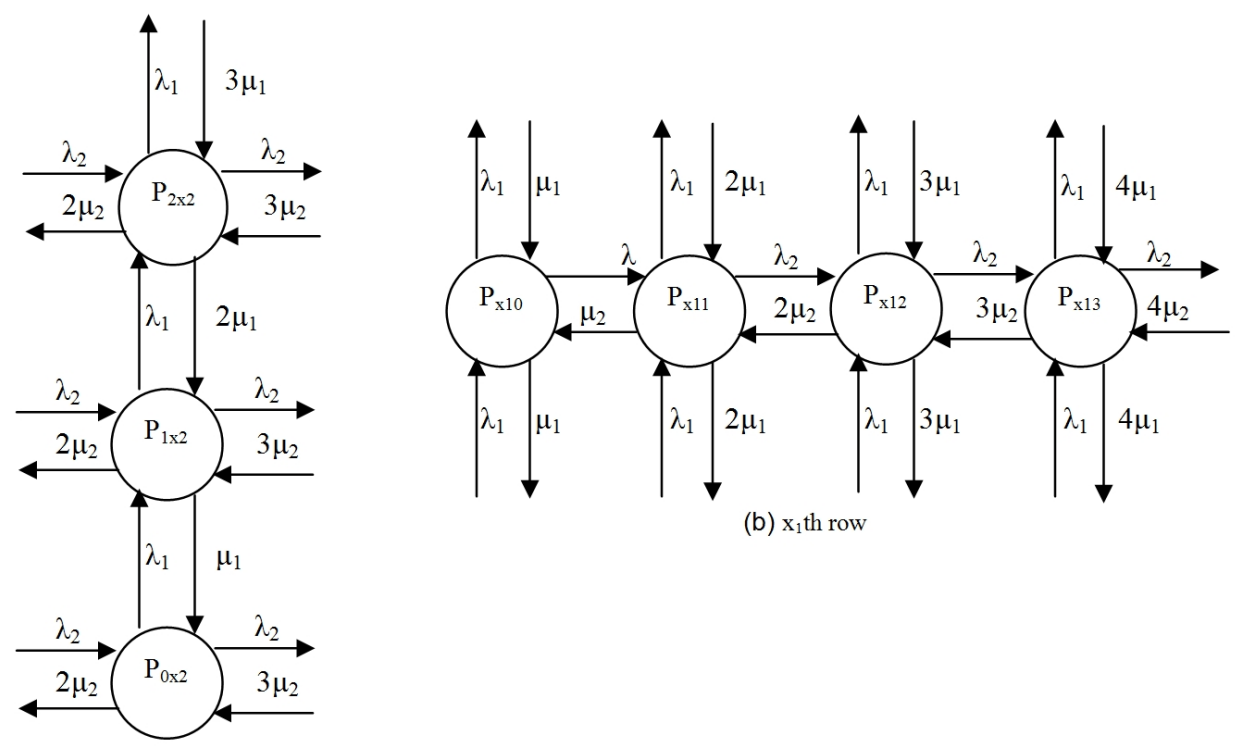

(b) $x_{1}$ th row

Fig. B.2. $x_{2}$ th column and $x_{1}$ th row of the 2-D chain

In generalized form:

$$
P_{x_{1} x_{2}}=\frac{A^{x_{1}} 1}{x_{1} !} P_{0 x_{2}}
$$

Again considering the $\mathrm{X}_{1}$ th row of Fig. B.2 (b) for cut equations.

Between the first and second node:

$P_{x 10} \lambda_{2}=P_{x 11} \mu_{2}$

$P_{x l, 1}=\left(\lambda_{2} / \mu_{2}\right) P_{x I 0}=A_{2} P_{x I 0}$

Between the second and third node:

$$
\begin{gathered}
\lambda_{2} P_{x_{1} 1}=P_{x_{1} 2} 2 \mu_{2} \\
P_{x_{1} 2}=\frac{\lambda_{2}}{2 \mu_{2}} P_{x_{1} 1}=\frac{A^{2} 2}{2 !} P_{x_{1} 0}
\end{gathered}
$$

In generalized form:

$$
P_{x_{1} x_{2}}=\frac{A_{2}^{x_{2}}}{x_{2} !} P_{x_{1} 0_{1}}
$$

Putting $\mathrm{x}_{2}=0$ in equation (B.1): 


$$
P_{x_{1} 0}=\frac{A^{x_{1}} 1}{x_{1} !} P_{00}
$$

From equation (B.2) and (B.3):

$$
P_{x_{1} x_{2}}=\frac{A_{2}^{x_{2}}}{x_{2} !} \frac{A_{1}^{x_{1}}}{x_{1} !} P_{00}
$$

Entire Probability State:

$$
\begin{aligned}
\sum_{x_{1}=0, x_{x}=0}^{\infty} P_{x_{x} x_{x}} & =1 \\
& \Rightarrow \sum_{x_{1}=0, x_{x}=0}^{\infty} \frac{A_{2}^{x_{2}}}{x_{2} !} \frac{A_{1}^{x_{1}}}{x_{1} !} P_{00}=1 \\
& \Rightarrow \sum_{x_{1}=0}^{\infty} \frac{A_{1}^{x_{1}}}{x_{1} !} \sum_{x_{2}=0}^{\infty} \frac{A_{2}^{x_{2}}}{x_{2} !} P_{00}=1 \\
& \Rightarrow\left(1+A_{1}+\frac{A_{1}^{2}}{2 !}+\frac{A_{1}^{3}}{3 !}+\ldots \ldots \ldots \ldots\right)\left(1+A_{2}+\frac{A_{2}^{2}}{2 !}+\frac{A_{2}^{3}}{3 !}+\ldots \ldots \ldots \ldots\right) P_{00}=1 \\
& \Rightarrow e^{A_{1}} \cdot e^{A_{2}} P_{00}=1 \quad \therefore P 00=e^{-(A 1+A 2)}
\end{aligned}
$$

From equation (B.4) and (B.5):

$$
P_{x_{1} x_{x}}=\frac{A_{1}^{x_{1}}}{x_{1} !} \frac{A_{2}^{x_{2}}}{x_{2} !} e^{-\left(A_{1}+A_{2}\right)}
$$

$\therefore$ For K-dimensional Poisson distribution:

$$
\begin{aligned}
P_{x_{1} x_{2} x_{3} \ldots \ldots \ldots x_{k}} & =\frac{A_{1}^{x_{1}}}{x_{1} !} \frac{A_{2}^{x_{2}}}{x_{2} !} \ldots \ldots \ldots \ldots . . . . \frac{A_{k}^{x_{k}}}{x_{k}} e^{-\left(A_{1}+A_{2}+\ldots \ldots \ldots+A_{k}\right)} \\
& =\prod_{i=1}^{k} \frac{A_{i}^{x_{i}}}{x_{i} !} e^{-A_{i}}
\end{aligned}
$$

When multidimensional Poisson traffic is offered to a limited number of servers $(\mathrm{n}<\propto)$ that are fully available to all traffic components (complete sharing), loss probabilities are calculated using the Erlang's multidimensional loss formula. For the $n$ Server case the entire probability states: 


$$
\begin{array}{r}
\sum_{x_{1}=0}^{n} \frac{A_{1}^{x_{1}}}{x_{1} !} \sum_{x_{2=0}}^{n-x_{1}} \frac{A_{2}^{x_{2}}}{x_{2} !} P_{00}=1 \\
\therefore P_{00}=\frac{1}{\sum_{x_{1}=0}^{n} \frac{A_{1}^{x_{1}}}{x_{1} !} \sum_{x_{2}=0}^{n-x_{1}} \frac{A_{2}^{x_{2}}}{x_{2} !}} \\
\text { Now: } P_{x_{1} x_{2}}=\frac{A_{1}^{x_{1}}}{\sum_{x_{1} !}^{n} \frac{A_{2}^{x_{2}}}{x_{2} !}} \frac{A_{1}^{x_{1}} \sum^{n-x_{1}} \frac{A_{2}^{x_{2}}}{x_{1} !}}{x_{2=0} !}
\end{array}
$$




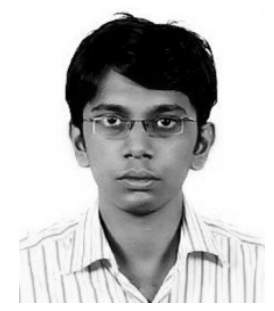

\section{Tonmoy Saha}

He received his B.Sc (Hons) in Computer Science \& Engineering from Jahangirnagar University, Savar, Dhaka, Bangladesh in 2008. He worked as a Lecturer in the Department of Computer Science and Engineering Department at Gono University, Savar, Dhaka, Bangladesh from May 20, 2010 to September 19, 2010. He is now working as a Software Engineer in the Advanced Research and Development Department in the Samsung Bangladesh R\&D Center Ltd. as of September 20, 2010. His research interests are Wireless communication, System on Chip (SOC), Real time Operating Systems (RTOS), Artificial Intelligence, Image Processing, and Software Engineering. He is now is pursuing his MS in Computer Science \& Engineering from Jahangirnagar University, Savar, Dhaka.

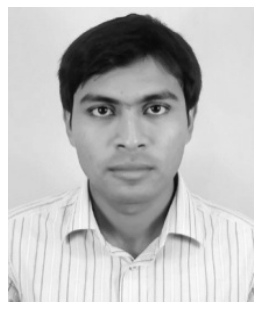

\section{Md. Abu Shufean}

He obtained his four-year Bachelor of Science with Honours degree in 2008 in Computer Science and Engineering from Jahangirnagar University, Savar, Dhaka, Bangladesh. The topics he is interested in are Artificial Intelligence, Mobile Communication, Software Engineering, Web Technology, and Programming. He is now working as a Software Engineer at SocialCube Ltd. Dhaka, Bangladesh. $\mathrm{He}$ is now pursuing his MS in Computer Science \& Engineering from Jahangirnagar University, Savar, Dhaka.

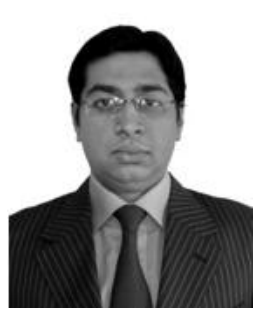

\section{Mahbubul Alam}

He completed his B.Sc. (Hons.) and MS in Computer Science and Engineering from Jahangirnagar University, Savar, Dhaka, Bangladesh in 2006 and 2008 respectively. He received the "Asadul Kabir Gold Medal" for securing the highest grade in the B.Sc. (Hons.) final examination 2006 at Jahangirnagar University. Since July 13, 2009 he has been working as a lecturer in the Department of Computer Science and Engineering at Jahangirnagar University, Savar, Dhaka, Bangladesh. His research interests are wavelet transform, wireless communications, adaptive filter theory, artificial intelligence, image processing, and machine learning.

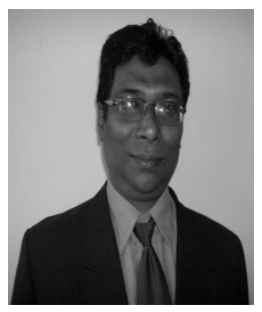

\section{Md. Imdadul Islam}

He completed his B.Sc. Engineering in Electrical and Electronic Engineering at Bangladesh University of Engineering and Technology, Dhaka in 1993 and his M.Sc Engineering from the same university in 1998. He has completed the CAS program at the Asian Institute of Technology in Thailand in 2001. He did his Ph.D. at the Department of Computer Science and Engineering, J.U., Dhaka, in the field of Network Traffic. He worked as an Assistant Engineer in Sheba Telecom (Pvt.) LTD (A joint venture company between Bangladesh and Malaysia) from Sept. '94 to July. '96. He has very good field experiences in the installation of Radio Base Stations and Switching Centers for WLL. He is now working as a professor in the Department of Computer Science and Engineering at Jahangirnagar University, Savar, Dhaka, Bangladesh. His research fields are network traffic, wireless communications, wavelet transform, OFDMA, WCDMA, adaptive filter theory, and array antenna systems. He has more than a hundred research papers in national and international journals and conference proceedings. 